\title{
De Revoluciones Y Reformas: tras un tipo ideal de la Reforma Social ${ }^{1}$
}

\author{
Raúl Enrique Rojo ${ }^{2}$
}

\section{Introducción}

De todas la teorías explicativas del cambio social, el marxismo fue, seguramente, aquella que pareció ofrecer más coherencia, más realismo y, al mismo tiempo, más esperanzas. Es desde esta perspectiva que la revolución apareció a un gran número de sociólogos, de historiadores y de politólogos como un momento privilegiado del cambio social. Había que esforzarse, así, por comprender la génesis, el estallido y los efectos de la revolución y sus efectos a corto y (sobre todo) a largo plazo. Es verdad que la historia de Occidente está marcada por ciertas revoluciones que produjeron una serie de efectos múltiples. El historiador norteamericano del

\footnotetext{
'La presente es una versión de la comunicación presentada en el XXYV Congreso de la Asociación Latinoamericana de Sociologia organizado en Arequipa (Perú) del 4 al 8 de noviembre de 2003, cuyo resumen publicó ese mismo año la Universidad Nacional de San Agustín de Arequipa en soporte digital (CD-ROM América Latina: hacia una nueva Alternativa de desarrollo). Paxa su redacción, el autor siguió de cerca el pensamiento de Guy Rocher, con quien tuvo el privilegio de trabajar durante el año académico boreal 2001-2002 en el CRDP de la Universidad de Montreal.

"Abogado y notario por la Universidad de Buenos Aires (UBA); Magíster en Sociología por la Pontificia Universidad Całólica Argentina "Santa María de los Buenos Aires" (UCA); Doctor en Sociología por la École des hautes études en sciences sociates (EHESS, París). Pusdoctor en Sociología Jurídica por la Université de Montréal (Quebec, Canadá), en la que se desempeñó como Profesor $e$ investigador visitante del Centre de recherche en droit public (CRDP). Ex docente de la Universidad de Buenos Aires. Director de investigaciones del Centre de recherches sur l'administration de la justice et la société (CRAJS, París). Profesor e investigador concursado de la Universidad Federal de Rio Grande do Sul (UFRGS, Porto Alegre), Programas de Posgrado en Sociología, Derecho y Relaciones Intemacionales. E-mail: raulrojo@vortex.ufrgs.br
} 
derecho Harold Berman identifica seis grandes revoluciones que han enmarcado la formación del derecho de los países occidentales. Las dos primeras son, en realidad, grandes reformas religiosas que modificaron profundamente la cristiandad occidental. Se trata, primero, de la Reformatio llevada a cabo, en buena parte, bajo la autoridad del Papa Gregorio VII, de donde viene el nombre de Reforma Gregoriana, y que Berman data con precisión entre 1075 y 1122. La segunda, fue el cisma que comenzó con los ataques de Lutero contra el Papado, que adoptó en Alemania el carácter de una "revolución nacional" y que es identificada generalmente como la "Reforma Protestante". Berman la sitúa entre 1517 y 1555. Que estas dos gtandes transformaciones de la cristiandad lleven el nombre de "reforma" oculta, según Berman, el hecho de que ambas han sido auténticas revoluciones por sus efectos a largo plazo, en particular sohre el derecho. Las otras cuatro revoluciones fueron políticas: la revolución británica de 1640-1688, conocida con el nombre de Glorious Revolution, y las revoluciones francesa, nortearnericana y rusa, que nos son generaimente más familiares (BERMAN, 1996: 29).

El interés de los sociólogos por las revoluciones fue singularmente grande durante los años 60 y 70 de la centuria pasada. Y si disminuyó por las razones que brillanternente expuso Norbert Lechner y que tan bien conocimos (si no padecimos) tantos intelectuales latinoamericanos (LECHNER, 1990: 17-27), no ha desaparecido, pese a todo. James Tilly ha escrito recientemente que "la construcción de modelos constantes (invariantes) de la revolución continúa siendo la actividad principal entre los sociólogos norteamericanos", una actividad que el propio Tilly encuentra una reverenda "pérdida de tiempo" (TILLY, 1995: 1605). Quizás por eso, a guisa de tespuesra a su crítica, se publicó después una recopilación de textos para testimoniat los progresos cumplidos en el análisis de las revoluciones y proponer nuevas hipótesis en la teorización de las mismas (FORAN, 1997).

El predominio (por no decir el tertorismo intelectual) que la teoría marxista del cambio social ejerció durante más de cuarenta años en el medio universitario (del cual la Asociación Latinoameticana de Sociología es uno de sus últimos reductos) y el interés que esa teoría demostró por las revoluciones proyectaron, como consecuencia, un cono de sombra sobre el papel desempeñado por las reformas sociales. Más aún, podriamos decir que la reforma goza de mala reputación: se la acusa de tener como finalidad y como efecto el oponerse a la revolución, de ser contrarrevolucionaria en el sentido literal del térnino. El reformismo ha sobtellevado así una carga senántica peyorativa, asociado como ha sido al conservadurismo y a la protección y defensa del statu quo y de los intereses a él asociados.

Esta manera de considerar toda reforma social, de desacreditarla, no carecía evidentemente de fundamentos reales. Es verdad que ciertas reformas pueden haber desempeñado este papel, a veces sin que sus promotores tuvieran conciencia de ello, pero muy a menudo de manera muy consciente. Sin embargo, el sociólogo que quiera comprender y explicar el cambio social no puede dejarse intimidar por estas 
consideraciones y evitar - como ocurrió muchas veces - el estudio de las reformas como fenómeno social y como agente de mudanzas sociales. En efecto, si bien es cierto que grandes mutaciones sociales han ocurrido como consecuencia de ciertas revoluciones, las reformas están rambién en el origen de muchas transformaciones sociales, económicas, políticas y culturales (y hasta podríamos decir que también espirituales). Su acción puede parecer más modesta, menos brillante o heroica; dan menos alas a la imaginación porque, generalmente más frías que las revoluciones, recurren menos a las pasiones y a las emociones.

Además, la gran variedad de las reformas es un motivo más para aumentar el interés que las mismas despiertan en el sociólogo. La reforma es, así, un vasto campo a desbrozar, empíricamente muy rico y muy diversificado. Ciertas reformas afectan al conjunto de una sociedad, otras se llevan a cabo en el interior de una institución (de una universidad, de un partido político o de un sindicato). La reforma puede venir "de arriba", prohijada por los dirigentes, para proyectarse después, poco a poco, a aquellos a quienes se supone que deben beneficiarse con ella. Pero una reforma puede también producirse desde las bases, puede ser pensada e inspirada por grupos (si no por grupúsculos) de ciudadanos insertados en el tejido social de la vida cotidiana, que hacen tomar conciencia de la necesidad y la urgencia de un cambio, que tratan de realizarlo ellos mismos, o intentan obligar a los dirigentes a aprobar y apoyar $s u$ reforma. Una reforma puede ser radical y expresar la intención de cambios profundos, aproximándose entonces al tipo de la revolución, con la cual frecuentemente será confundida. Pero puede también proponer mudanzas moderadas y sectoriales que no cuestionen las insrituciones en su conjunto.

La floresta de una "sociología de las reformas" es inmensa y sigue más o menos virgen (si algo puede ser "virgen a medias", con el debido respeto al abbé Prévosr). Y lo que es peor, a la penuria de estudios empíricos sobre las reformas sociales se sigue una la lamentable pobreza en la reflexión sociológica sobre este tema. La revolución ha dado lugar a vastas obras de síntesis, fruto del trabajo de historiadores, sociólogos y cientistas políticos, e inspiró a numerosos filósofos. La reforma social está lejos de haber gozado de una atención similar.

La historia occidental de estos últimos cincuenta años nos ha convencido, sin embargo, de la importancia de las reformas sociales para entender la realidad que nos circunda, para comprender por qué y cómo ciertas cosas han ocurrido. Por eso, habiéndonos interesado desde hace mucho tiempo, como sociólogos (y debo decir que también como ciudadanos), al fenómeno social de las reformas, hemos creído útil hacer de ellas el objeto de este trabajo. 


\section{La evolución de la noción de reforma}

La noción de reforma exige ser aclarada. La historia del término es ya esclarecedora de por sí. El Diccionario manual e ilustrado de la lengua española de la Real Academia nos dice que el verbo "reformar", de origen latino, significa, en primer lugar, "volver a formar... reparar, restaurar, restablecer, reponer". Por su parte, el Diccionario ideológico de Julio Casares nos recuerda que el "re", en "teformar", "señala una vuelta al pasado". Siendo aún más específico, este léxico agtega que el verbo indica la acción de "reducir [a alguien o a alguna cosa] a una forma mejor, a un estado preferible, siendo generalmente concebida esta forma como más antigua o primitiva". El sentido original del verbo "reformar" es entonces el de un retorno a unas maneras, ciertos valores o a una forma de vida anteriores, considerados preferibles a los que vivimos ahora, que se perdieron con el tiempo y que habría que revivir o a los cuales es preciso volver para ser mejores, para hacer mejor nuestra existencia o mejorar nuestras condiciones de vida.

Definida de esra forma, no puede extrañarnos que la intención de reformat hunda sus raíces históricas en el universo de las instituciones eclesiásticas cristianas, particularmente en el universo eclesiástico medieval, cuando se trata (como enseña el Diccionario enciclopédico bispanoamericano) de "teducir o restituir una orden religiosa u otro instituto a su primitiva observancia o disciplina" volviendo al espíritu y a la letra de la regla original. En la esfera general de la vida religiosa, la vida monacal es particularmente exigente, impone renuncias que a veces es difícil aceptar y hacer respetat. Se hacen, entonces, progresivamente, algunas concesiones, se instala una cierta "rutina" $y$, como consecuencia, pueden sobrevenir determinadas formas de relajación de las costumbres. Es cuando aparece un reformador, un San Benito, un San Bernardo de Clairvaux, un Papa Gregorio VII que viene a recordar las exigencias olvidadas, un poco a la manera en que lo hacían los grandes profetas de Istael, que recordaban a los judíos su Alianza con Yhaveh, de la cual se habían apartado con culposa indiferencia.

En la bistoria cristiana de Occidente, el término "Reforma" iba a tomar un sentido idéntico, pero en un nuevo contexto (a raíz de la mal llamada Reforma Protestante), que consistía en oponerse a la Iglesia Romana en nombre de un supuesto retorno a las verdaderas fuentes del cristianismo. Asi se instiruye una Iglesia (presuntamente) Reformada, inspirada por Calvino, convirtiéndose el adjetivo "reformado" en sustantivo para designar a un protestante. La expresión "reformarse" toma entonces una significación muy específica para querer indicar "pasarse al protestantismo", "modificar las reglas del cristianismo", es decir: hacerse protestante. La reacción de la Iglesia Católica a la Reforma Protestante adopró diversas formas, pero los historiadores modernos (haciéndole un flaco favor) le dieron el nombre de "Contrarreforma" (cuando en realidad ella era la verdadera reforma, operada para hacer frente al cisma protestante) (ROJO, 1982). 
Fue necesario un viraje increßble para que el término moderno "reforma" no designase más un retorno al pasado, una vuelta a las fuentes, sino un movimiento de avance, un cambio inspirado por un proyecto de futuro visto como una mejora, sin invocar necesariamente un pasado inspirador. Es posible que esta migración de sentido se haya operado, por una parte, a través del sustantivo francés "réformation" (y sus equivalentes en otras lenguas romance) de uso común durante mucho tiempo en las cortes europeas, especialmente en el lenguaje juridico y sobre todo político. Se hablaba a mediados del siglo XIV de la "réformation du royaume" para designar un programa político destinado a acabar con las grandes crisis sociales, económicas y políticas y, de esa manera, mejorar el estado del reino de Francia y la condición de su población (DEMURGER, 1990: 21). El término "réformation" pasó a las ottas lenguas neolatinas (dando "reformación" en castellano, "riformazione" en italiano y "reformaçấo" en portugués) y cayó en desuso en ellas a partir del siglo XVII en favor de reforma. Sin embargo habrá que esperar hasta el siglo XIX para que la noción de "reforma social" sea formulada con un sentido político, en Inglaterra, Francia y los Estados Unidos, para designar una intención no revolucionaria, aunque más o menos radical, de cambio social.

En realidad, los dos términos, el de "reforma" y el de "revolución", han conocido el mismo "giro semántico". De acuerdo a su sentido original, la revolución, como la reforma, expresa un movimiento circular, un retorno al punto de partida. Es la que, siguiendo a François Furet, podemos llamar de "idea astronómica de revolución" (FURET, 1985). Cuando decimos que el año es el periodo de tiempo que transcurre durante una "revolución" real de la Tierra en su órbita alrededor del Sol, aludimos a este concepto de revolución como regreso al punto de partida. Es así como ciertos comentadores de la época veían a la Glorious Revolution, no como la transformación radical que del pasado haria table rase (según la idea que los jacobinos franceses se hicieron de su propia revolución, y que evoca una estrofa de L'Internationale), sino como un retorno a las "viejas buenas leyes de los leales barones" que habían sido desvirtuadas por los "malvados monarcas" (los Estuardo) y sus partidarios jacobitas (ROJO, 2000). Fue preciso un importante cambio de sentido para que la revolución llegase a describit las transformaciones sociales y políticas profundas, llevadas a cabo en un riempo relativamente corto, como resultado de acciones multitudinarias y acompañadas de un cierto grado de violencia (SKOCPOL, 1985).

Hay, así, un cierto parentesco y, al mismo tiempo, una cierta oposición entre las nociones de reforma y de revolución. Es, por lo demás, comparando y oponiendo una a otra que podemos obtener una noción más clara y formular la mejor definición de ambas, en particular de la reforma que es el objeto de nuestra presente reflexión. Si consideramos en primer lugar la revolución, la misma se manifiesta por una transformación rápida y brusca, que marca una clara solución de continuidad y una transgresión de los límites de la legalidad existente, con la intención de producir un cambio total, fundamental. La acompaña, generalmente, un período más o menos 
largo de luchas, de violencia y aún de guerra, y trae aparejada una serie de efectos de largo alcance en el tiempo (BERMAN, 1996: 29 y KAHN, 1999: 46).

Comparada a esa descripción de la revolución, la teforma se presenta como un cambio más lento, escalonado en el tiempo, inspirado por una volunrad de modificar las cosas de una manera gradual, manteniendo una cierta continuidad con la situación actual, situación que se quiere pese a todo mejotar, pero dentro de la legalidad existente. Los efectos producidos por la reforma deben ser reales y efectivos, pero son de una naturaleza generalmente pragmática y de un alcance más medio que largo en el tiempo.

Esta caracterización de la reforma, comparada a la revolución, precisa aún de ciertas precisiones. La primera se refiere a su relación con el derecho. Una y otra, la revolución y la reforma, traen aparejadas importantes transformaciones del derecbo. Podríamos decir que sus efectos se inscriben en un nuevo derecbo. Sin embatgo, en el caso de la revolución, ésta comienza por una transgresión al derecbo existente y se concluye por una suerte de refundición fundamental del derecho público preexistente, generalmente mediante una nueva Constitución que reformula sobre nuevas bases la concepción y las estructuras del Estado y sus relaciones con los ciudadanos. La reforma comienza y se prosigue, al contrario, en el matco de la legalidad existente, aportándole no obstante los cambios necesarios para realizar e institucionalizar las intenciones del proyecto de reforma.

En segundo lugat, la revolución y la reforma se orientan, en principio, tanto una como la otra, de cara al futuro más que al pasado. Ambas pretenden generalmente hallarse inspiradas más por un proyecto innovador que por el retorno a un pasado supuestamente mejor. Una y otra necesitan, entonces, al principio sobre todo, una descripción y una evaluación negativas del presente estado de cosas, que es necesatio cambiar a la luz de una reflexión y de promesas a la vez consrructivas y proactivas. En la práctica, sin embargo, las cosas no son ran claras y traslúcidas. La reforma, en particular, puede ser en esre aspecto más ambigua que la revolución. Puede ser propio de ella mezclar en un mismo proyecto los recuerdos de un pasado añorado con ideas que se pretenden innovadoras. Hay, así, reformas que se han inspirado más en el pasado que en el futuro.

Un tercer elemento muy importante se refiere a la legitimidad que una y otra necesitan. Grandes cambios sociales no se producen sin que sus promotores basen su acción en algún tipo de legitimidad. La revolución, como se produce en la ilegalidad, encuentra primero su legitimidad en la claridad del proyecto propuesto y en el carisma personal de los jefes que la inspiran, y después en la eficacia real de los cambios logrados. En cuanto a la reforma, subsistirá como legítima en la medida en que, al contrario de la revolución, su proyecto se mantenga dentro de la legalidad vigente y pueda prometer cumplirse y producir efectos gracias a modificaciones bien 
enmarcadas en la legislación vigente y en continuidad con ella. El fundamento racional de la legitimidad de la revolución y de la reforma aparece, entonces, como esencialmente diferente a pesar de ser absolutamente necesario para ambas.

Subrayemos, a mayor abundamiento, que el análisis sociológico de la revolución y de la reforma debe poner énfasis sobre el hecho de que ambas se llevan a cabo por la acción de actores singularmente diversos. Dejo aquí voluntariamente de lado este aspecto de la revolución que Theda Skocpol ha caracterizado con Lujo de detalles. En lo que concierne a la reforma, ésta implica la acción diversificada de numerosos actores. Unos actuando como los inspiradores de la reforma, otros como promotores, mientras que otros más se convierten en los realizadores. Puede verse entonces cómo se constituye rápidamente una jerarquía que define el estatus y el rol de cada categoría de actores, así como las formas de comunicación y las relaciones de autoridad entre ellos. Estas relaciones no están exentas de tensiones (a menudo muy grandes) entre los diferentes niveles de autoridad y de poder, tensiones que se producen tanto como consecuencia de las concepciones que cada uno tiene de los objetivos de la reforma, como de las formas concretas de realización de la misma. Frente a estos actores comprometidos con la reforma, se erigen los actores que se oponen a la reforma. Estos opositores pueden ser adversarios activos que denuncien y busquen derrotar la reforma, pero a menudo también se encuentra un número más grande de opositores pasivos, cuya inacción reviste diversas formas (generalmente eficaces) para vaciar de efectos la reforma, minimizarlos o cuando menos reducir su alcance.

Finalmente, la reforma, como la revolución, debe tener efectos, aportar cambios suficientemente numerosos y durables, que se extiendan durante un periodo de tiempo suficientemente prolongado. Una reforma no es tal si no produce un efecto. De lo contrario, será un proyecto de reforma, una intervención, la utopia, quizás, de una reforma. Pero los histotiadores no se referirán a ella como una reforma. Sin embargo, el efecto principal, procurado tanto por la revolución como por la reforma, es un nuevo consenso en el seno de la sociedad, un equilibrio más estable en las relaciones de poder entre las clases, los estratos, las categorías y los grupos de ciudadanos.

La exposición precedente, haciendo nuestra la metodología de Max Weber, ha procurado elaborar lo que podriamos denominar el "tipo puro" o el "tipo ideal" de la reforma, comparado al de revolución. Este tipo ideal, por el conjunto de rasgos que vincula entre sí, hace las veces de una definición. En la realidad empírica, lo que interesa al sociólogo es, precisamente, la correspondencia de los casos reales con el modelo, al mismo tiempo que las diferencias con el mismo. Así, es la relación de fuerzas entre todas las categorias de actores la que permititá que el proyecto reformista se concretice o fracase y que la versión original del mismo tenga que someterse a concesiones más o menos importantes o que llegue a su fin tal como había sido concebido.

Siguiendo esta metodologia, llevamos adelante un proyecto de investigación 
que consiste en aprehender, de la manera más precisa posible, la noción de reforma social, para permitirnos, de seguido, embarcarnos en el análisis de ciertas reformas que constituyen los procesos identificados como la "Reforma Universitaria" argentina de 1918 (particularmente de sus avatares durante el período 1957-1966); la "Revolución Tranquila" quebequense de 1960-1970 y la "Movida" española de 1982-1992.

Este trabajo empirico está en plena ejecución: hemos cumplido, así, una primera fase teórica y levantado los primeros datos en la Argentina y el Quebec, por lo que suponemos que será acabado "in situ", en España, en 2004. El avance de nuestra investigación permite ya, a la luz del material recopilado y del análisis comparado de los casos argentino y quebequense, poner de resalto algunos elementos que perrenecen, según nos parece, al tipo ideal de las reformas y que se deducen del análisis sociológico que estamos realizando.

Elementos fundadores de una teorización de la reforma

Se trata de ciertos rasgos y caracteres que pueden servir de elementos fundadores de una teorización de la reforma. Los presentaremos siguiendo cuatro ejes.

\section{EjeI-Efectividad ylegitimidad}

En primer lugar, debemos volver sobre una consideración anterior recordando que, para ser reconocida a posteriori como tal por el análisis histórico, una reforma tiene que tener efectos. Debe haber cambiado el estado de cosas imperante, se trate de instituciones, costumbres, tradiciones o mentalidades (por separado o al mismo tiempo). Esto es, tiene que tener "efectividad".

Eso quiere decir que la noción de innovación es inherente a la de reforma. Una innovación que puede incluir un cierto retorno al pasado pero que no deja de proyectarse al futuro. La reforma implica innovación en el sentido de que supone la intención exitosa de modificar la realidad vigente y de introducir en ella un elemento activo de transformación.

La efectividad de la reforma, es decir su aptitud para producir efectos, se basa en otro elemento esencial para toda reforma, a menudo ignorado: su legitimidad. La hemos evocado antes y volvemos a insistir sobre ella por que se trata de un elemento del cual poco se dice, ya que generalmente se da por supuesto que toda reforma siempre se basa en una cierra legirimidad. La misma puede, sin embargo, ser más compleja de lo que se piensa y revestir diversos aspectos. Evidentemente, toda reforma que venga "de arriba", es decir que sea promovida por una autoridad en ejercicio (política o religiosa, según el caso), se apoya en una legitimidad de tipo jurídico. Una autoridad anuncia, emprende y lleva a cabo una reforma en virtud de poderes que el derecho vigente le confiere. No obstante, se puede observar empíricamente que, si esta legitimidad jurídica es comunmente admitida al comienzo, puede empezar a ser 
contestada a lo largo del proceso de reformas, en la medida en que el mismo comience a sufrir tropiezos, se empantane, o produzca indeseables efectos secundarios. Se podrá recusar, entonces, la legitimidad jurídica en nombre de una legitimidad que podríamos llamar sociológica: es así, por ejemplo, que los docentes o los padres de alumnos (o los dos a la vez) pueden cuestionar la legitimidad de un ministerio de educación "reformista" como consecuencia de los "errores" que se le atribuyan; o que los profesionales de la salud (médicos, enfermeras y personal auxiliar) pueden deslegitimar a los funcionarios y burócratas gubernamentales a raíz de su supuesto desconocimiento de las condiciones reales de la práctica de la medicina y de la vida de hospital que pretendían mudar. La legitimidad jurídica sobre la que se pueden apoyar los reformadores no es, así, inagotable: aún cuando no sea cuestionada en el plano estrictamente legal, tiene que contar con un cierto éxito para ser completada y reforzada por la legitimidad sociológica. Esta última es menos visible y mucho más discreta que la jurídica pero puede, pese a todo, adquirir una fuerza tal como para minar eficazmente la legitimidad jurídica e impedir el avance de las reformas. La legitimidad jurídica habilita a los actores para emprender una reforma, pero la legitimidad sociológica les aporta la credibilidad necesaria para llevarla a cabo.

La reforma que viene "de abajo", de las bases, plantea problemas de legitimidad absolutamente diferentes. Generalmente no se basa, en sus comienzos, en una legitimidad jurídica. A diferencia de la revolución, una reforma promovida por las bases resta en los límites de la legalidad, pero eso no le confiere una legitimidad específica. Se podría decir que una reforma de este tipo obtiene su legitimidad del discurso de sus jefes o portavoces y de lo que podriamos llamar junto con Max Weber el "carisma de la palabra" (WEBER, 1992: 864). Hay que entender los discursos de los jefes no tanto como una predicción sino como la descripción de la movilización necesaria para hacer el cambio posible, factible y deseable. La legitimidad carismática permite lanzar la idea de una reforma y emprenderla. Pero en la medida que la idea gane adherentes, el proyecto de reforma deberá institucionalizarse en una asociación, un movimienro o un partido. La legitimidad carismática, si continúa viva, tiene que ser asociada a una legitimidad sociológica, primero, y después, cada vez más, a una legitimidad jurídica.

\section{Eje II-Los actores y la construcción de la reforma}

El análisis de los diferentes tipos de legitimidad de la reforma nos conduce a estudiar los actores comprometidos en toda reforma. Determinar con precisión las diversas categorías de actores implicados en las mismas y las múltiples interacciones que se dan entre ellos es una dimensión esencial de la inteligibilidad de los procesos que nos propusimos estudiar. Es preciso establecer, en particular, una clara distinción entre los primeros actores que han idealizado una reforma, de aquellos que a continuación la pusieron en marcha y se esforzaron por llevarla a cabo. Estos últimos, los realizadores, se encuentran incluidos, frecuentemente, en una escala jerárquica 
que parte de los que planifican, ordenan y coordinan, pasa por los que a continuación traducen los proyectos en directivas, normas y reglas, y culmina en los que realizan concretamente la reforma (en las agencias del Estado, las aulas, los hospitales, las fábricas o los cuarteles). A ellos debemos agregar los actores que se oponen a la reforma en diferentes niveles, que la contestan, la retardan o la hacen desviar de sus objetivos. El análisis de las estrategias de estos diferentes actores, de las justificaciones que ellos invocan, de las significaciones que dan a sus acciones, de las relaciones de fuerza y de poder que se establecen entre ellos, nos abre un inmenso "laboratorio" de observación empírica y de reflexión teórica.

Es tomando en cuenta el punto de vista de los actores, sus diversas "perspectivas" sobre la reforma y el "significado" que los mismos acuerdan a los hechos y a los ottos actores, que hemos comenzado a estudiar provechosamente los tres casos de reforma antes citados como un constructo, como una construcción artificial resultado de la diversidad de los actores. Esta problematización "constructivista" de las reformas es esencial para el análisis sociológico. Toda reforma ha sido hecha por actores y ella es el resultado no sólo de quienes la iniciaron, sino también de todos los actores que participaron en su realización y de todos aquellos que se opusieron a la misma. Bajo la acción de estos diversos actores, la reforma se construye y reconstruye constantemente, obedeciendo a distintas estrategias y a los múltiples intereses de todos los grupos y de todas las categorías de actores.

\section{Eje III-Latraducción normatiuade Lareforma}

Para ser completo y esclarecedor, este análisis de la "reforma en acción" a través de los diferentes actores debe desembocar en el estudio de las diversas normativas (jurídicas o de otra especie) y de las relaciones de los actores con aquellas regulaciones. Una reforma se realiza en parte por la traducción de sus objetivos y de su ideología en directivas, reglamentaciones, normas y reglas. Algunas son estrictamente legislativas y jurídicas, otras son administrativas y técnicas, otras en fin son estrictamente sociológicas, resultado espontáneo de las necesidades de la acción de los diferentes actores. Estos ordenamientos se convierten en la expresión institucional de los objerivos de la reforma y sirven tanto de objetivos en las estrategias de los actores como de marco a sus acciones e interacciones. Estos cuerpos de normas pueden, de acuerdo a las circunstancias, racionalizar las relaciones de fuerza entre los actores o, al contrario, hacerlas más duras favoreciendo ciertos grupos de actores en perjuicio de ottos.

\section{EjeIV-Reformay clase media}

Una última consideración: si la reforma, tal como acabamos de caracterizarla, se ha convertido en un modo corriente de innovación y cambio social, es evidentemente porque la misma se ha adaptado al tipo democtático de sociedad. Más precisamente, ello se debe a que la reforma responde al espíritu y a la mentalidad de la clase media. 
Resumiremos brevemente esta mentalidad diciendo que ella se caracteriza por el individualismo y la búsqueda de realización personal, una actitud consumista al encuentro de todos los bienes y servicios posibles, una movilización política sectorial orientada por inrereses privados y una alergia a toda forma de pensamiento y de acción radicales.

Esta clase se ha vuelto hoy hegemónica en las sociedades democráticas modernas aprovechando la declinación demográfica y política de la clase obrera y la falencia intelectual de su idearió revolucionario. Porque la revolución no está en el ánimo de la clase nedia: demasiados intereses la ligan a las estructuras económicas, sociales y políticas de las modernas sociedades capitalistas. Sin embargo, ella también tiene interés en que estas estructuras y sus instituciones funcionen mejor y rindan más. Las reformas que apoya la clase media tienen generalmente por objetivo mejorar el funcionamiento y la eficacia de las instituciones vigentes y favorecer así la prosperidad general, de la cual todos esperan, de acuerdo con el individualismo imperante, sacar provecho.

De ahí se siguen dos consecuencias importantes. En primer lugar, tiende a desaparecer la diferencia entre las reformas que vienen "de abajo" y las que se generan "arriba". Las sociedades democráticas de las clases medias han instalado un tipo de gobernanza en el que se encuentran y ponen de acuerdo, cada vez más, los representantes del Estado y los de los diversos intereses de la sociedad civil. La toma de decisiones se produce en los puntos de intersección entre el Estado y la sociedad civil. Los idealizadores de las reformas que vienen "de arriba" se esfuerzan por lograr que los grupos de interés implicados compartan sus objetivos, valiéndose para ello de diversos modos de "democracia participativa". Este es un aspecto esencial del gobierno democrático de acuerdo al espiritu de la clase media. Sobre una suerte de continuum que podríamos representar gráficamente y que iría de un polo representado por las transformaciones que vienen más clatamente de la autoridad política estatal, hasta otro polo que concentraria las demandas más claramente sentidas por los ciudadanos, las reformas tienden actualmente a ubicarse en el centro, resultado de proyectos provenientes "de arriba" y de propuestas articuladas "desde abajo".

La segunda consecuencia consiste en una disminución constante de la distancia semántica existente entre revolución y reforma. Las auténticas revoluciones son raras, cada vez inás räras, mientras que las reformas, en cambio, se hacen cada vez más numerosas y casi permanentes. En este contexto, las reformas de envergadura son declaradas "tevoluciones". El lenguaje corriente ha hecho suya esta evolución, ya que se recurre comunmente a los términos "revolución" y "revolucionario" para 
designar los cambios sociales transaccionales y sus alcances. Es así que las cinco reformas de los años 1960-1970 en el Quebec se han convertido en la "Revolución Tranquila", y que se conoce por "Revolución Verde" al conjunto de transformaciones en el campo de la agricultura que fueron propiciadas por la FAO y que los posteriores avances en ingeniería genética acabaron por profundizar ${ }^{3}$. Por otro lado, ha aparecido una nueva terminología que da cuenta de la misma evolución en sentido inverso: se habla de "transición" para designar el pasaje de los regímenes autoritarios latinoamericanos y de las sociedades comunistas de Europa Central y Oriental a regímenes democráticos y formas de sociedad liberal.

Proponemos hacer coincidir aquí estas consideraciones con otras hechas hace más de un siglo y medio por Alexis de Tocqueville, con las cuales deseamos concluir.

\section{Conclusiones}

En el segundo tomo de La democracia en América, que apareció en 1840, cinco años después del primero, Tocqueville formuló un gran número de reflexiones de naturaleza especificamente sociológica. Es interesante leer, entre otros, el capítulo XXI tirulado "Porqué las grandes revoluciones serán cada vez menos frecuentes". Sobre este tema Tocqueville escribe: "No es que yo diga que las naciones democráticas estén al abrigo de revoluciones: digo ran sólo que el estado social de esas naciones no las predispone a ellas, sino que mas bien las aleja". Tocqueville se exphca esta situación por la igualdad social creciente y el individualismo que la acompaña, dos evoluciones inherentes a la sociedad democrática, que conducen a los ciudadanos a replegarse "en el círculo de los menudos intereses domésticos". En consecuencia, concluye el perspicaz Tocqueville, "es de prever que acabarán por hacerse inaccesibles a esas grandes y poderosas emociones públicas que turban a los pueblos, pero que los alimenran y los renuevan... no puedo menos de temer que los hombres lleguen al punto de considerar toda nueva teoría como un peligro, toda innovación como un enojoso desorden, todo progreso social como un primer paso hacia una revolución... Tengo miedo, lo confieso, de que se dejen dominar hasta tal punto por un miserable gusto por los goces del día, que su interés por el propio fururo y el de sus descendientes desaparezca y prefieran seguir muellemente el curso de su destino a hacer, si es preciso, un súbito y enérgico esfuerzo para enderezarlo. Sé que las nuevas sociedades cambiarán cada día su aspecto, pero me temo que acabarán fijándose con excesiva indiferencia en las mismas instituciones, los mismos prejuicios y las mismas costumbres, de suerte que el desarrollo humano se frene... y que la humanidad, removiéndose sin

${ }^{3}$ Y recordemos que, ya en los años de 1970 , un partido de centro como el Demócrata Cristiano chileno propuso una "revolución en libertad". 
cesar, no dé un paso adelante" (TOCQUEVILLE, 1980: 218 y 224).

Podrá extrañar, quizás, encontrar en la pluma de Tocqueville palabras tan favorables a la revolución y su miedo de asistir a la desaparición de las condiciones favorables a su estallido. Con la perspectiva que nos acuerdan los años podemos decir hoy que esta predicción de Tocqueville se ha cumplido parcialmente y que las democracias modernas parecen, efectivamente, haber perdido el fermento revolucionario. Pero esto no significa, sin embargo, que se hayan estabilizado tanto como lo pretendía nuestro autor. Es que éste no pudo prever ni el papel que iba a desempeñar la clase media ahora dominante (como medio de identificación o aún de referencia) en las democracias modernas, ni las reformas sociales que la misma favorece o acepta, particularmente las que vienen "de arriha", y que han reemplazado a las revoluciones para satisfacer los anhelos de cambio social. Es que, en los hechos, la igualdad social está lejos de haberse convertido en realidad como lo pretendía Tocqueville. Estudiar como sociólogo las reformas sociales más que las revoluciones, es entonces proseguir, adaptándolo a nuestra época, el pensamiento de Alexis de Tocqueville sobre los caminos (atajos y rodeos) del cambio social. Creemos que es una buena brújula para nuestro periplo. 


\section{BIBLIOGRAFIA}

BERMAN, Harold J. 1996. La formación de la tradición jurídica de Occidente, México, Fondo de Cultura Económica.

DEMURGER, Alain. 1990. Temps de crises, temps d'espoirs, XIVe-XVe siècle, París, Seuil.

FORAN, John (compilador). 1997. Theorizing revolutions, Nueva York, Routledge.

FURET, François. 1985. Penser la Révolution française, París, Gallimard, Collection Folio/ Histoire.

LECHNER, Norbert 1990. Lospatios interiores de la democracia Subjetividady política, Santiago [Chile], Fondo de Cultura Económica.

ROCHER, Guy. 2001. Le "laboratoire" des réformes dans la Révolution tranquile. Montreal, Programme d'études sur le Québec de l'Université McGill.

ROJO, Raúl Enrique. 1982. "El reino en Roger Bacon y Dante Alighieri", Revista de la Universidad Católica de La Plata, III, 8, pp. 121-147.

ROJO, Raúl Enrique. 2000. Confessionalismo político e escola republicana, Porto Alegre, manuscrito.

SKOCPOL, Theda. 1985. Etats et révolutions sociales. La révolution en France, en Rusie et en Chine, París, Fayard.

TILLY, James. 1995. “To explain political processes", American Journal of Sociology, 180, 6, pp. 1605-1629.

TOCQUEVILLE, Alexis de. 1980. La democracia en América, Madrid, Alianza Editorial, t. 2 .

WEBER, Max. 1992. Economía y sociedad. Esbozo de sociología comprensiva. Buenos Aires, Fondo de Cultura Económica. 